\title{
Does Celiac Disease Affect Antibody Production Against Hepatitis B in
} Children?

\author{
Fatemeh Dastmalchi ${ }^{1}$, Mehri Najafi (iD) ${ }^{2,3}$, Parisa Rahmani ${ }^{1,2,3}$, Kambiz Eftekhari (iD ${ }^{2,3}$, Pejman Rohani ${ }^{4}$ \\ and Hosein Alimadadi (iD) $1,2,3,{ }^{*}$ \\ ${ }^{1}$ Children's Medical Center, Pediatrics Center of Excellence, Tehran, Iran \\ ${ }^{2}$ Pediatric Gastroenterology and Hepatology Research Center, Tehran University of Medical Sciences, Tehran, Iran \\ ${ }^{3}$ Department of Pediatrics, Tehran University of Medical Sciences, Tehran, Iran \\ ${ }^{4}$ Pediatric Gastroenterology and Hepatology Research Center, Mofid Children’s Hospital, Tehran, Iran \\ "Corresponding author: Children's Medical Center, Pediatrics Center of Excellence, Tehran, Iran. Tel: +98-2161472094, Email: hoseinalimadadi@yahoo.com
}

Received 2020 February 24; Revised 2020 May 19; Accepted 2020 June 26.

\begin{abstract}
Background: Celiac disease is an immune-mediated systemic disease, in which gluten ingestion causes different symptoms. HLADQ2 is positive in $90 \%-95 \%$ of celiac patients. HLA has been considered to inhibit antibody production against the hepatitis B virus vaccine. Considering that celiac disease affects about $1 \%$ of the population, this phenomenon could be a significant factor in immunization programs. On the other hand, HLA-DQ2 positive patients not only are at the risk of HBV infection themselves but also have the potential to be an important source of HBV dissemination in the world.

Objectives: This study was carried out to evaluate the responsiveness of celiac-affected children to HBV vaccination in Iran.

Methods: This case-control prospective study was performed on 62 Iranian children ( 31 children with confirmed celiac disease before introducing a gluten-free diet and 31 healthy children). HBS Ab (antibody against hepatitis B virus surface antigen) titer was checked in the patients and compared between the two groups.

Results: It was shown that $67.7 \%$ of cases and $64.5 \%$ of controls had HBS Ab titer above $10 \mathrm{mIU} / \mathrm{mL}$, but the difference was not significant statistically. After matching for the time interval from the last HBV vaccination, it was observed that although non-significantly, celiac disease can decrease the chance for anti-HBS production up to $30 \%$.

Conclusions: This study did not confirm non-responsiveness against the HBV vaccine in celiac disease in children. This may be due to genetic factors or type of vaccine. Moreover, in our study, responsiveness was assessed qualitatively in the two groups (HBS Ab > $10 \mathrm{mIU} / \mathrm{mL}$ was considered as a responder).
\end{abstract}

Keywords: Children, Celiac Disease, HBV Vaccine

\section{Background}

Celiac disease is a multifactorial gluten-sensitive autoimmune enteropathy (1). It is recognized in the background of specific HLA-DQ2 or HLA-DQ8 and high levels of antibodies (anti-tissue transglutaminase 2 antibody or Anti-TG2 IgA and anti endomysial antibody or EMA) according to different clinical findings, and in gluten-consuming children according to pathologic findings $(2,3)$.

Celiac disease is relatively frequent, with the incident rate of about $0.7 \%-1.4 \%$ in most populations (4). Based on the 2012 ESPGHAN (European Society of Pediatric Gastroenterology, Hepatology and Nutrition) guideline, the diagnosis of celiac disease can be confirmed by pathological changes Marsh II or III in the background of high anti-TG2 ab OR anti-TG2 10-folds above the normal values plus a positive EMA antibody in the presence of HLA-DQ2/HLA-DQ8 in symptomatic children (3).

Hepatitis B is an important health problem in the world $(5,6)$. Fortunately, an effective and safe vaccine against HBV has been introduced since 1982. Currently, all children are immunized against this infection all over the world (7). The trend of health strategies is to eradicate this infection. A rate of $90 \%-100 \%$ of vaccinated people whose anti-HBS ab titer is above $10 \mathrm{mIU} / \mathrm{ml}$ are protected against HBV infection $(7,8)$.

1.1. Different Factors Are Responsible for Unresponsiveness Against HBV Vaccine

Inappropriate environment of vaccine maintenance, bad site of injection, obesity, cigarette smoking, drug abuse, and some infections. Moreover, alcohol consumption, renal insufficiency, HIV infection, immune suppres- 
sion, and diabetes mellitus are associated with less antibody production (9-11).

HLA-DQ2 is positive in diseases such as celiac and diabetes mellitus. This HLA is associated with lower titers of HBS ab $(12,13)$. The gluten consumption during HBV vaccination in celiac-affected children might affect the production of HBS ab $(14,15)$. Other studies describe that both HBS Ag and gliadin peptides have an affinity for HLA-DQ2; therefore, these two peptides attempt to connect to HLA-DQ2 molecule, resulting in an incomplete antibody response against HBS Ag (15).

These celiac disease-affected children are a significantly large population that can be potentially vulnerable to hepatitis B, and more importantly, they can be a major source of HBV dissemination in the world. This is a critical issue because it can damage the health care system by lowering the vaccination program's efficiency.

\section{Objectives}

This study was designed to determine the rate of responsiveness to $\mathrm{HBV}$ vaccination (anti-HBS Ab titer $>10$ $\mathrm{mIU} / \mathrm{mL}$ ) in a group of Iranian children with celiac disease.

\section{Methods}

In this cross-sectional case-control study, 31 Iranian children with celiac disease were enrolled as the case group, and 31 age- and sex-matched non-celiac children in the same period were included in the control group.

\subsection{Inclusion Criteria}

Every child with celiac disease (based on high antiTG2 ab and duodenal biopsy results, suggesting Marsh II or III, according to our center's local protocol in the absence of reliable HLA typing laboratories) was included in our case group before introducing a gluten-free diet. Our control group consisted of children without celiac disease, other autoimmune disorders, and well-known immunodeficiency (primary or secondary).

\subsection{Exclusion Criteria}

Included:

Proven immune deficiency,

Lack of anti-HBV vaccination history,

Use of immunosuppressives during the last three months,

History of transfusion, dialysis, or plasmapheresis during the last three months.

The setting of the study was in Children's Medical Center, a tertiary level referral hospital in Tehran, Iran. There was no ethical consideration, Anti-HBS Ab test was done free in all patients after obtaining informed consent and assuring confidentiality for the parents/guardians. The serum sample of celiac-affected children before introducing a gluten-free diet and control group children was analyzed for HBS ab titer using the Abbot chemiluminescence kit. Anti-HBS Ab titer above $10 \mathrm{mIU} / \mathrm{mL}$ was considered positive. Other data including age, sex, BMI, other concomitant diseases such as diabetes mellitus and autoimmune thyroiditis, time after last HBV vaccination, and anti-TG2 titer were recorded using a questionnaire. We used $t$-test as well as McNamara and Fisher tests for analysis. The data were analyzed with SPSS software version 20. A P value of less than 0.05 was considered statistically significant.

\section{Results}

In this study, 31 celiac-affected children aged 24 - 180 months were compared with 31 non-celiac age- and sexmatched children.

\subsection{Case Group}

The average age of the cases was $81.84 \pm 39.54$ months (72 months for boys and 85 months for girls). The HBS ab titer was below $10 \mathrm{mIU} / \mathrm{mL}$ in $44.4 \%$ of the boys and $27.6 \%$ of the girls. However, there was no significant difference between boys and girls $(\mathrm{P}=0.35)$. Moreover, the difference between the boys and girls was not significant, with regard to concomitant autoimmune disease $(P=0.29)$.

The average age of vaccine responders and nonresponders was 72 and 100 months, respectively, and the difference was marginally significant $(\mathrm{P}=0.06)$.

The vaccine responsiveness rate in children younger than 45 months, between 45 - 60 months, between 60 - 114 months, and older than 114 months was 74\%,100\%, 70\%, and $37.5 \%$, respectively. The Fisher test revealed no significant difference $(\mathrm{P}=0.109)$ in this regard.

Based on the $t$-test, the time interval from the last HBV vaccination was longer, but not significantly, in the nonresponders ( 94.7 months vs 66.7 months, $\mathrm{P}=0.06$ ).

The average level of serum Anti-TG2 Ab in the responders and non-responders was $186 \mathrm{U} / \mathrm{mL}$ and $163.5 \mathrm{U} / \mathrm{mL}$, respectively, and the difference was not significant $(\mathrm{P}=0.2)$. The summary of the data is shown in Table 1.

\subsection{Case and Control}

A rate of $67.7 \%$ of the cases and $64.5 \%$ of the controls had HBS ab titer above $10 \mathrm{mIU} / \mathrm{mL}$. Bi-variable analysis using the McNemar test showed no statistically significant difference $(P=0.73)$ between the two groups. However, 


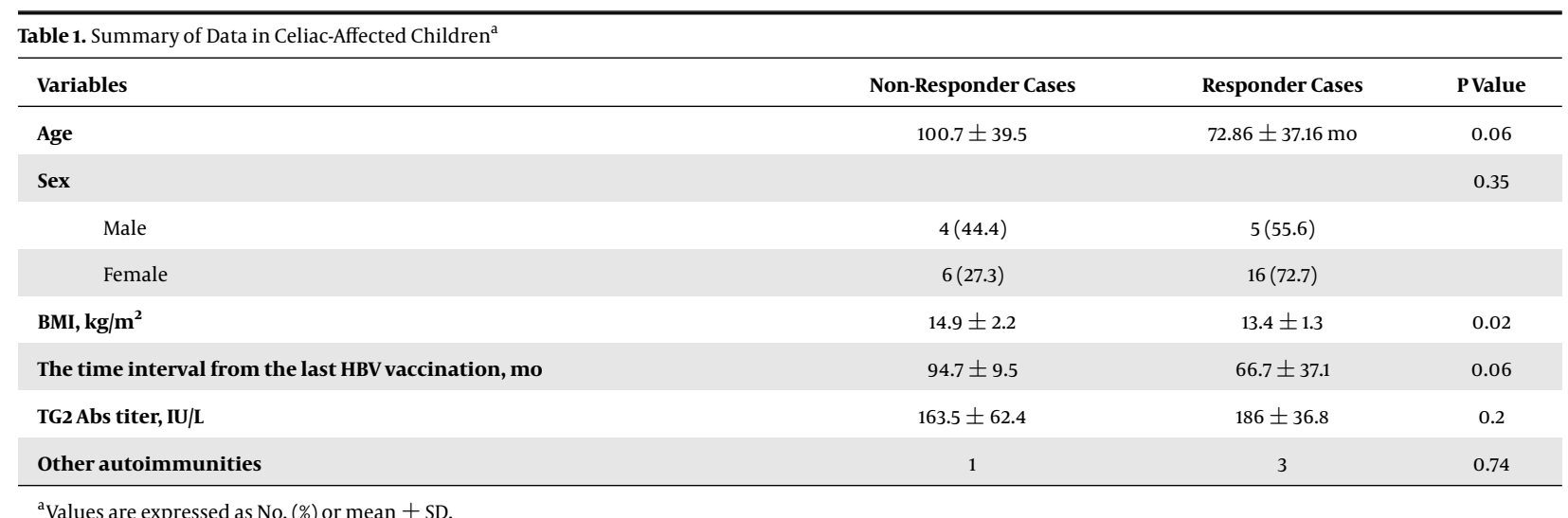

the odds ratio was 0.8 , meaning that, although non significantly, celiac disease decreases the antibody production against HBV by up to $20 \%$ compared to the control group.

Conditional regression analysis showed that the matched odds ratio for comparing HBS ab titer between the case and control groups was 0.7 (7.26 and 0.07; CI: 95\%). After matching for the time interval from the last HBV vaccination, it was observed that although non-significantly, celiac disease can decrease the chance for anti-HBS production up to $30 \%$. The comparison result of the two groups is shown in Table 2.

\begin{tabular}{|c|c|c|}
\hline Variables & Control Group & Case Group \\
\hline Age & $81.87 \pm 37.97$ & $81.84 \pm 39.54$ \\
\hline \multicolumn{3}{|l|}{ Sex } \\
\hline Female & $22(71)$ & $22(71)$ \\
\hline Male & $9(29)$ & $9(29)$ \\
\hline BMI, $\mathrm{kg} / \mathrm{m}^{2}$ & $30.4 \pm 16.9$ & $13.9 \pm 1.76$ \\
\hline Responsiveness to HBV vaccine & $20(64.5)$ & $21(67.7)$ \\
\hline $\begin{array}{l}\text { The time interval from the last } \mathrm{HBV} \\
\text { vaccination, mo }\end{array}$ & $162 \pm 75.71$ & $174 \pm 75.84$ \\
\hline Other autoimmuneities & 0 & $4(12.9)$ \\
\hline Diabetes mellitus & 0 & $3(9.7)$ \\
\hline Autoimmune thyroiditis & 0 & $1(3.2)$ \\
\hline
\end{tabular}

${ }^{\mathrm{a}}$ Values are expressed as No. (\%) or mean \pm SD.

\section{Discussion}

HBV infection is one of the most important health problems in the world, and vaccination is the best way of prophylaxis (4). The effective blood level of antibody (HBS ab level $>10 \mathrm{mIU} / \mathrm{mL}$ ) is achieved by a three-dose cycle of
HBV vaccination in 95\% of children (8). Moreover, 90\% $100 \%$ of people with effective HBS ab levels are protected against $\mathrm{HBV}$ infection (7).

Moreover, $4 \%-10 \%$ of vaccinated people do not produce sufficient HBS ab in response to the vaccine, probably because of bad vaccine maintenance conditions, obesity, inappropriate vaccination site (e.g., gluteal), smoking, chronic alcoholism, chronic renal insufficiency, immune deficiency, and other causes (9-11).

HLA-DQ2 is positive in more than $90 \%$ of celiac patients (1). This HLA is associated with lower titers of HBS ab (12, 13). Noh et al. (12), in a case-series study in 2003, found that 13 out of 19 celiac patients did not have protective levels of HBS antibodies, and all were HLA-DQ2 positive. Some other studies also showed that antibody production was lower in celiac patients than in non-celiac ones $(13,14,16$ 18). Ertekine et al. (17) suggested that there should be a distinct vaccination protocol for celiac patients. Zanoni et al. (18) concluded that these different responsiveness might be related to different intervals from the last HBV vaccination. In our study, this was not confirmed by matching the cases regarding this variable.

Nemes et al. (15) and Leonardi et al. (14) suggested that gluten consumption was a probably important factor in antibody production against the HBV vaccine. However, this was not affirmed in our study, and anti-HBS ab levels were analyzed before introducing a gluten-free diet.

In a systematic review and meta-analysis by Opri et al. (19) in 2015, 12 prospective studies (1447 patients) and four retrospective studies (184 patients) were reviewed. The non-responsiveness rate was $47 \%$ and $36 \%$ in celiac patients in prospective and retrospective studies, respectively. The difference between celiac and non-celiac patients was statistically significant. They suggested that in celiac diseaseaffected children, it be better to determine anti-HBS ab levels as soon as possible. Further, they concluded that a personalized anti-HBV vaccination schedule and a follow-up 
program were necessary for celiac patients (19).

We used a qualitative method to evaluate responsiveness to the HBV vaccine, anti-HBS ab titers more than 10 $\mathrm{mIU} / \mathrm{mL}$ were considered as responsiveness, which may have affected our study results. Thus, a quantitative study is recommended for assessing the effects of celiac disease on antibody production against HBV vaccine.

\subsection{Conclusions}

This study did not confirm non-responsiveness against the HBV vaccine in celiac disease in children. This may be due to genetic factors or type of vaccine. Moreover, in our study, responsiveness was assessed qualitatively in the two groups (HBS Ab > $10 \mathrm{mIU} / \mathrm{mL}$ was considered as a responder).

\section{Footnotes}

Authors' Contribution: Hosein Alimadadi conceptualized and designed the study and wrote and submitted the manuscript. Mehri Najafi conceptualized and designed the study. Fatemeh Dastmalchi conceptualized and designed the study, collected the data, analyzed and interpreted the data, and wrote as a thesis. All authors revised and approved the final manuscript and accepted to be included in all aspects of this study.

Conflict of Interests: All authors declare that they have no conflict of interest.

Ethical Approval: All procedures performed in the study were in accordance with the ethical standards of the local Ethics Committee of Tehran University of Medical Sciences and with the 1964 Helsinki declaration and its later amendments or comparable ethical standards. Ethics approval code was (Ethics Committee of Tehran University of Medical Sciences): IR.TUMS.VCR.REC.1395.614.

Funding/Support: This study was not supported by any university or company or research center.

Informed Consent: Informed consent was obtained from all individual participants included in the study.

\section{References}

1. Ronald EK, Olivier-Jean G, Giorgina M, Ian RS, Philip MS. Walker's pediatric gastrointestinal disease: Pathology, diagnosis, management. 6th ed. USA, Ltd: PMPH; 2008.

2. Robert K, Joseph S. Nelson textbook of pediatrics. 21th ed. Elsevier; 2020.

3. Husby S, Koletzko S, Korponay-Szabo IR, Mearin ML, Phillips A, Shamir R, et al. European Society for Pediatric Gastroenterology, Hepatology, and Nutrition guidelines for the diagnosis of coeliac disease. J Pediatr Gastroenterol Nutr. 2012;54(1):136-60. doi: 10.1097/MPG.0b013e31821a23do. [PubMed: 22197856].
4. Singh P, Arora A, Strand TA, Leffler DA, Catassi C, Green PH, et al. Global prevalence of celiac disease: Systematic review and meta-analysis. Clin Gastroenterol Hepatol. 2018;16(6):823-836 e2. doi: 10.1016/j.cgh.2017.06.037. [PubMed: 29551598].

5. Frederick JS, Ronald JS, William FB. Liver disease in children. 4th ed. Cambridge: Cambridge University Press; 2014.

6. Ahmadinejad Z, Abdi Liae Z, Salehizadeh S, Mansori S, Alijani N. Efficacy of post-exposure prophylaxis in infants born to hbsag positive mothers in Iran; is it authentic? Iran J Pediatr. 2016;26(3). e5979. doi 10.5812/ijp.5979. [PubMed: 27617077]. [PubMed Central: PMC4992177].

7. Andre FE. Overview of a 5-year clinical experience with a yeast-derived hepatitis B vaccine. Vaccine. 1990;8 Suppl:S74-8. discussion S79-80. doi: 10.1016/0264-410x(90)90222-8. [PubMed: 2139288].

8. Da Villa G, Pelliccia MG, Peluso F, Ricciardi E, Sepe A. Anti-HBs responses in children vaccinated with different schedules of either plasma-derived or HBV DNA recombinant vaccine. Res Virol. 1997;148(2):109-14. doi: 10.1016/s0923-2516(97)89893-7. [PubMed: 9108609].

9. Sjogren MH. Prevention of hepatitis B in nonresponders to initial hepatitis B virus vaccination. Am J Med. 2005;118 Suppl 10A:34S-9S. doi 10.1016/j.amjmed.2005.07.012. [PubMed: 16271539].

10. Yu AS, Cheung RC, Keeffe EB. Hepatitis B vaccines. Infect Dis Clin North Am. 2006;20(1):27-45. doi: 10.1016/j.idc.2006.01.004. [PubMed: 16527647].

11. Yang S, Tian G, Cui Y, Ding C, Deng M, Yu C, et al. Factors influencing immunologic response to hepatitis B vaccine in adults. Sci Rep. 2016;6:27251. doi: 10.1038/srep27251. [PubMed: 27324884]. [PubMed Central: PMC4914839].

12. Noh KW, Poland GA, Murray JA. Hepatitis B vaccine nonresponse and celiac disease. Am J Gastroenterol. 2003;98(10):2289-92. doi: 10.1111/j.1572-0241.2003.07701.x. [PubMed: 14572581].

13. Park SD, Markowitz J, Pettei M, Weinstein T, Sison CP, Swiss SR, et al. Failure to respond to hepatitis $B$ vaccine in children with celiac disease. J Pediatr Gastroenterol Nutr. 2007;44(4):431-5. doi: 10.1097/MPG.0b013e3180320654. [PubMed: 17414139].

14. Leonardi S, Spina M, Spicuzza L, Rotolo N, La Rosa M. Hepatitis B vaccination failure in celiac disease: Is there a need to reassess current immunization strategies? Vaccine. 2009;27(43):6030-3. doi: 10.1016/j.vaccine.2009.07.099. [PubMed: 19682619].

15. Nemes E, Lefler E, Szegedi L, Kapitany A, Kovacs JB, Balogh M, et al Gluten intake interferes with the humoral immune response to recombinant hepatitis $\mathrm{B}$ vaccine in patients with celiac disease. Pediatrics. 2008;121(6):e1570-6. doi: 10.1542/peds.2007-2446. [PubMed: 18519462].

16. Ertem D, Gonen I, Tanidir C, Ugras M, Yildiz A, Pehlivanoglu $\mathrm{E}$, et al. The response to hepatitis B vaccine: Does it differ in celiac disease? Eur J Gastroenterol Hepatol. 2010;22(7):787-93. doi: 10.1097/MEG.0b013e32832e9d41. [PubMed: 19584738].

17. Ertekin V, Tosun MS, Selimoglu MA. Is there need for a new hepatitis B vaccine schedule for children with celiac disease? Hepat Mon 2011;11(8):634-7. doi: 10.5812/kowsar.1735143x.715. [PubMed: 22140387]. [PubMed Central: PMC3227494].

18. Zanoni G, Contreas G, Valletta E, Gabrielli O, Mengoli C, Veneri D. Normal or defective immune response to Hepatitis B vaccine in patients with diabetes and celiac disease. Hum Vaccin Immunother. 2015;11(1):58-62. doi: 10.4161/hv.34309. [PubMed: 25483516]. [PubMed Central: PMC4514307].

19. Opri R, Veneri D, Mengoli C, Zanoni G. Immune response to Hepatitis B vaccine in patients with celiac disease: A systematic review and meta-analysis. Hum Vaccin Immunother. 2015;11(12):2800-5. doi: 10.1080/21645515.2015.1069448. [PubMed: 26378476]. [PubMed Central: PMC5054794] 